\title{
Clinical, pathological, and PAM50 gene expression features of HER2-low breast cancer
}

\author{
Francesco Schettini $\mathbb{D}^{1,2,3}$, Nuria Chic ${ }^{2,3,4}$, Fara Brasó-Maristany ${ }^{2,4}$, Laia Paré $\mathbb{i D}^{3}$, Tomás Pascual (iD) $2,3,4,5$, Benedetta Conte ${ }^{2,6}$, \\ Olga Martínez-Sáez ${ }^{2,4}$, Barbara Adamo ${ }^{2,3,4}$, Maria Vidal ${ }^{2,3,4}$, Esther Barnadas ${ }^{2}$, Aranzazu Fernández-Martinez ${ }^{5}$, \\ Blanca González-Farre ${ }^{2,3,7}$, Esther Sanfeliu ${ }^{2,3,7}$, Juan Miguel Cejalvo ${ }^{8}$, Giuseppe Perrone ${ }^{9}$, Giovanna Sabarese ${ }^{9}$, Francesca Zalfa ${ }^{9}$, \\ Vicente Peg $^{10,11}$, Roberta Fasani ${ }^{12}$, Patricia Villagrasa ${ }^{3}$, Joaquín Gavilá ${ }^{3,13}$, Carlos H. Barrios (D) ${ }^{14,15}$, Ana Lluch (iD) ${ }^{11,16,17}$, Miguel Martín ${ }^{11,18}$, \\ Mariavittoria Locci ${ }^{19}$, Sabino De Placido ${ }^{1}$ and Aleix Prat $\mathbb{I D}^{2,3,4 凶}$
}

Novel antibody-drug conjugates against HER2 are showing high activity in HER2-negative breast cancer (BC) with low HER2 expression (i.e., $1+$ or $2+$ and lack of ERBB2 amplification). However, the clinical and molecular features of HER2-low BC are yet to be elucidated. Here, we collected retrospective clinicopathological and PAM50 data from 3,689 patients with HER2-negative disease and made the following observations. First, the proportion of HER2-low was higher in HR-positive disease (65.4\%) than triplenegative BC (TNBC, 36.6\%). Second, within HR-positive disease, ERBB2 and luminal-related genes were more expressed in HER2-low than HER2 0. In contrast, no gene was found differentially expressed in TNBC according to HER2 expression. Third, within HER2-low, ERBB2 levels were higher in HR-positive disease than TNBC. Fourth, HER2-low was not associated with overall survival in HR-positive disease and TNBC. Finally, the reproducibility of HER2-low among pathologists was suboptimal. This study emphasizes the large biological heterogeneity of HER2-low BC, and the need to implement reproducible and sensitive assays to measure low HER2 expression.

npj Breast Cancer (2021)7:1 ; https://doi.org/10.1038/s41523-020-00208-2

\section{INTRODUCTION}

HER2-positive breast cancer is currently defined according to the ASCO/CAP guidelines using immunohistochemistry (IHC) and/or in situ hybridization (ISH)-based techniques ${ }^{1,2}$. These guidelines identify a tumor as HER2-positive when there is a complete and intense circumferential HER2 IHC staining in $\geq 10 \%$ of cells (score $3+$ ) and/or the gene is amplified with an HER2/CEP17 ratio $\geq 2.0$ and an average HER2 gene (ERBB2) copy number $\geq 4.0$ signals/cell using ISH-based techniques ${ }^{1}$. In breast cancer, $10-20 \%$ of tumors are HER2-positive and $80-90 \%$ are HER2-negative ${ }^{3,4}$.

Within HER2-negative disease, substantial heterogeneity exists regarding the expression of hormone receptors (HR) and HER2. For example, HER2-negative tumors can express some protein level of HER2 by $\mathrm{IHC}^{5}$ (i.e., $1+$ or $2+$ and lack of ERBB2 amplification by in situ hybridization techniques) and are identified as HER2-low. Traditionally, patients with HER2-low-expressing tumors do not seem to benefit from HER2-targeted therapies, such as 1-year of adjuvant trastuzumab ${ }^{6}$. However, two HER2-directed antibodydrug conjugates (ADC) with chemotherapeutics, namely trastuzumab deruxtecan (T-DXd) and trastuzumab duocarmazine (SYD985) have shown very promising therapeutic activity in patients with HER2-low breast cancer $^{7-9}$. A large pivotal randomized phase III trial of T-DXd in patients with pretreated HER2-low metastatic breast cancer is underway (i.e., NCT03734029/DESTINY-Breast04).
Owing to the recent and increased interest in the HER2-low group, there is an urgent need to better understand its clinicopathological and molecular features. Thus, we decided to collect clinicopathological and PAM50 gene expression data from multiple datasets ${ }^{10-17}$ of HER2-negative disease and compare many features between HER2-low and HER2 0. Analyses were focused on the overall population and according to hormone receptor (HR) status and HER2 IHC expression.

\section{RESULTS}

Clinicopathological characteristics of HER2-low disease

Thirteen independent datasets for a total of 3,689 patients with HER2-negative breast cancer were explored (Fig. 1). Overall, 1,486 (40.3\%) patients had HER2 0 tumors, 1,489 (40.4\%) had HER2 1+ tumors and 714 (19.3\%) had HER2 2+ tumors. Clinicopathological and gene expression data (when available) were largely obtained from primary disease $(71.1 \%$ in HER2-low and $73.7 \%$ in HER2 0). According to HR status, 2,962 (80.8\%) patients had HR-positive disease and 706 (19.2\%) had triple-negative breast cancer (TNBC).

HER2-low tumors were more frequently found within HRpositive disease compared to TNBC $(65.4 \%$ vs. $36.5 \%, p<0.001$; Fig. 2). More specifically, HR-positive disease was characterized by higher rates of IHC $1+$ and $2+$ tumors, compared to TNBC $(43.8 \%$

\footnotetext{
Department of Clinical Medicine and Surgery, University of Naples Federico II, Naples, Italy. ${ }^{2}$ Translational Genomics and Targeted Therapeutics in Solid Tumors, August Pi Sunyer Biomedical Research Institute, Barcelona, Spain. ${ }^{3}$ SOLTI Breast Cancer Research Group, Barcelona, Spain. ${ }^{4}$ Department of Medical Oncology, Hospital Clínic, Barcelona, Spain. ${ }^{5}$ Department of Genetics, University of North Carolina, Chapel Hill, NC, USA. ${ }^{6}$ Department of Medical Oncology, Ospedale Policlinico San Martino, University of Genova, Genova, Italy. ${ }^{7}$ Department of Pathology, Hospital Clínic, Barcelona, Spain. ${ }^{8}$ INCLIVA Biomedical Research Institute, Hospital Clínico Universitario Valencia, University of Valencia, Valencia, Spain. ${ }^{9}$ Pathology Department, Campus Bio-Medico University, Rome, Italy. ${ }^{10}$ Vall d'Hebron University Hospital, Barcelona, Spain. ${ }^{11}$ GEICAM, Grupo Español de Investigación en Cáncer de Mama, Madrid, Spain. ${ }^{12}$ Vall d'Hebron Institute of Oncology (VHIO), Barcelona, Spain. ${ }^{13}$ Instituto Valenciano de Oncología (IVO), Valencia, Spain. ${ }^{14}$ Centro de Pesquisa Clínica Hospital São Lucas da PUCRS, Porto Alegre, Brazil. ${ }^{15}$ LACOG, Latin American Cooperative Oncology Group, Porto Alegre, Brazil. ${ }^{16}$ Hospital Universitario Clínico Valencia, Valencia, Spain. ${ }^{17}$ Biomedical Research Centre Network in Cancer (CIBERONC), Valencia, Spain. ${ }^{18}$ Hospital Gregorio Marañon, Madrid, Spain.

${ }^{19}$ Department of Neuroscience, Reproductive Sciences and Dentistry, University of Naples Federico II, Naples, Italy. ${ }^{凶}$ email: alprat@clinic.cat
} 


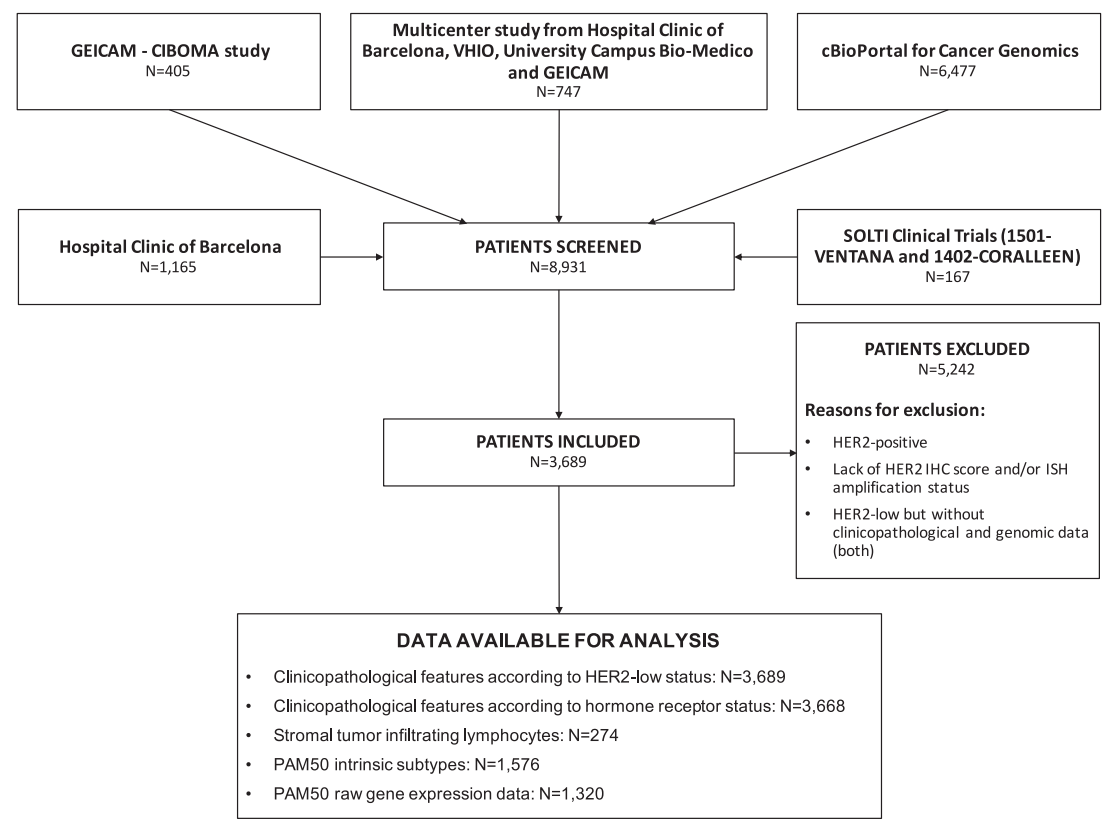

Fig. 1 STROBE flow-chart. Flow-chart resuming the patient selection process, showing causes for exclusion and the number of patients with available data for the main analyses presented in the study. GEICAM Grupo Español de Investigación en Cáncer de Mama, CIBOMA Coalición Iberoamericana de Investigación en Oncología Mamaria, VHIO Vall d'Hebron Institute of Oncology, SOLTI Solid Tumor Intensification Group, IHC immunohistochemistry, ISH in-situ hybridization, HR hormone receptors.
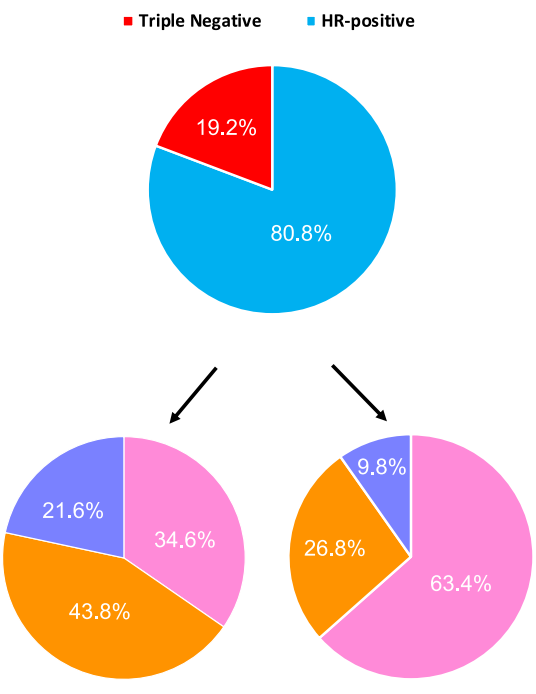

$=\mathrm{HHCO}=\mathrm{IHC} 1+\quad-\mathrm{IHC} 2+$
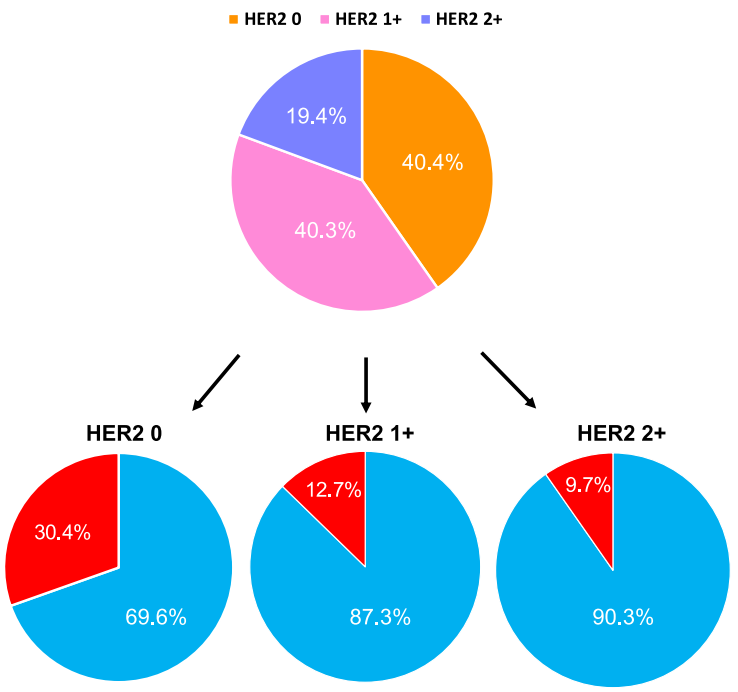

- HR-positive $\quad$ Triple Negative

Fig. 2 Hormone receptor status, HER2-low status, and IHC scores distributions within the HER2-negative population. HR hormone receptors, IHC immunohistochemistry, ISH in situ hybridization (including either FISH, SISH, and CISH).

vs. $26.8 \%$ and $21.6 \%$ vs. $9.8 \%$, respectively, $p<0.001$; Fig. 2 ). In terms of other clinicopathological variables, HER2-low tumors presented larger primary tumor sizes $(p=0.007)$ and more nodal involvement $(p=0.010)$ compared to HER2 0 tumors (Table 1 and Supplementary Table 1). No male patient was observed within the HER2 0 cohort, compared to the 15 cases observed in the HER2low subset $(p=0.001)$. The median age at diagnosis was higher for the HER2-low tumors compared to HER2 0 (59 vs. 55 years, $p=$ $0.003)$. No statistically significant differences were observed in terms of menopausal status $(p=0.898)$, histological grade $(p=$ 0.175 ), Ki67 IHC scores ( $p=0.092$ using a 14\% cut-off) and percentage of stromal tumor-infiltrating lymphocytes (TILs) $(p=$ 0.218), although TILs' levels were differently distributed according to HER2 IHC levels $(p=0.033)$ and were higher in HER2 $2+$ (median: 5; interquartile range [IQR] 1-5) compared to $1+$ (median: 1 ; IQR $1-5 ; p=0.035$ ) and 0 (median: $1 ;$ IQR $1-5 ; p=$ 0.035).

Reproducibility of the HER2-low classification

To evaluate the reproducibility of HER2 IHC scoring among pathologists, we scanned 200 HER2 IHC stained slides from 100 independent cases of the Hospital Clinic case series. The images were representative of the $4 \mathrm{HER} 2 \mathrm{IHC}$ categories (i.e., $0,1+, 2+$ and $3+$ ). Five breast cancer-specialized pathologists (BG, ES, RF, $\mathrm{GP}$, and VP), coming from four different institutions (Clinic, VHIO, VHV, and Campus Bio-Medico), revised and scored the 100 cases 


\begin{tabular}{|c|c|c|c|c|c|c|c|}
\hline \multirow[t]{3}{*}{ Demographics } & \multicolumn{6}{|c|}{ HER2-negative } & \multirow[t]{3}{*}{$p^{\mathrm{a}}$} \\
\hline & \multicolumn{2}{|c|}{ HER2 0} & \multicolumn{2}{|c|}{ HER2-low } & \multicolumn{2}{|c|}{ Overall population } & \\
\hline & 1,486 & 40.3 & 2,203 & 59.7 & 3,689 & 100 & \\
\hline \multicolumn{8}{|l|}{ Age at diagnosis (years) } \\
\hline Min-max & $24-93$ & & $26-96$ & & $24-96$ & & \\
\hline Pts with available data & 259 & 27.4 & 685 & 72.6 & 944 & 100 & \\
\hline \multicolumn{8}{|l|}{ Sex } \\
\hline Male & 0 & 0 & 15 & 0.7 & 15 & 0.4 & 0.001 \\
\hline Female & 1,486 & 100 & 2,187 & 99.3 & 3,673 & 99.6 & \\
\hline Total & 1,486 & 40.3 & 2,202 & 59.7 & 3,688 & 100 & \\
\hline \multicolumn{8}{|l|}{ Biospecimen } \\
\hline Primary lesion & 1,000 & 73.7 & 1,382 & 71.1 & 2,382 & 72.1 & 0.096 \\
\hline Other lesion & 357 & 34.6 & 563 & 28.9 & 920 & 27.9 & \\
\hline Total & 1,357 & 41.1 & 1,945 & 58.9 & 3,302 & 100 & \\
\hline \multicolumn{8}{|l|}{ Histotype } \\
\hline Ductal & 639 & 70.8 & 1,214 & 74.3 & 1,853 & 73 & 0.175 \\
\hline Lobular & 194 & 21.5 & 314 & 19.2 & 508 & 20 & \\
\hline Other & 69 & 7.6 & 107 & 6.5 & 176 & 6.9 & \\
\hline Total & 902 & 35.6 & 1,635 & 64.4 & 2,537 & 100 & \\
\hline \multicolumn{8}{|l|}{$\mathrm{T}$} \\
\hline 1 & 509 & 55.8 & 807 & 48.7 & 1,316 & 51.2 & 0.007 \\
\hline 3 & 46 & 4.9 & 135 & 8 & 181 & 6.9 & \\
\hline Total & 945 & 35.9 & 1,684 & 64.1 & 2,629 & 100 & \\
\hline \multicolumn{8}{|l|}{ ER } \\
\hline Positive & 983 & 67 & 1,894 & 87.1 & 2,877 & 79 & $<0.001$ \\
\hline Negative & 484 & 33 & 280 & 12.9 & 764 & 21 & \\
\hline Total & 1,467 & 40.3 & 2,174 & 59.7 & 3,641 & 100 & \\
\hline \multicolumn{8}{|l|}{$\mathrm{PgR}$} \\
\hline Positive & 789 & 54.7 & 1,542 & 71.8 & 2,331 & 64.9 & $<0.001$ \\
\hline Negative & 654 & 45.3 & 606 & 28.2 & 1260 & 35.1 & \\
\hline Total & 1,443 & 40.2 & 2,148 & 59.8 & 3,591 & 100 & \\
\hline G & & & & & & & \\
\hline 1 & 67 & 8.8 & 139 & 10.6 & 206 & 9.9 & 0.0499 \\
\hline 2 & 272 & 35.6 & 514 & 39.1 & 786 & 37.8 & \\
\hline 3 & 426 & 55.7 & 660 & 50.3 & 1086 & 52.3 & \\
\hline Total & 765 & 36.8 & 1,313 & 63.2 & 2,078 & 100 & \\
\hline Ki67 & & & & & & & \\
\hline Median & 16 & & 18 & & 18 & & 0.892 \\
\hline IQR & $9-30$ & & $10-27$ & & $10-27$ & & \\
\hline Min-max & $0.5-95$ & & $0.5-95$ & & $0.5-95$ & & \\
\hline Pts with available data & 433 & 36.4 & 756 & 63.6 & 1,189 & 100 & \\
\hline$\leq 14 \%$ & 190 & 43.9 & 294 & 38.9 & 484 & 40.7 & 0.092 \\
\hline$>14 \%$ & 243 & 56.1 & 462 & 61.1 & 705 & 59.3 & \\
\hline$<20 \%$ & 236 & 54.5 & 411 & 54.4 & 647 & 54.4 & 0.963 \\
\hline$\geq 20 \%$ & 197 & 45.5 & 345 & 45.6 & 542 & 45.6 & \\
\hline
\end{tabular}




\begin{tabular}{|c|c|c|c|c|c|c|c|}
\hline \multirow[t]{3}{*}{ Demographics } & \multicolumn{6}{|c|}{ HER2-negative } & \multirow[t]{3}{*}{$p^{\mathrm{a}}$} \\
\hline & \multicolumn{2}{|c|}{ HER2 0} & \multicolumn{2}{|c|}{ HER2-low } & \multicolumn{2}{|c|}{ Overall population } & \\
\hline & 1,486 & 40.3 & 2,203 & 59.7 & 3,689 & 100 & \\
\hline \multicolumn{8}{|l|}{ TILs } \\
\hline Min-max & $0-80$ & & $0-80$ & & $0-80$ & & \\
\hline Pts with available data & 102 & 37.2 & 172 & 62.8 & 274 & 100 & \\
\hline \multicolumn{8}{|l|}{ PAM50 subtypes } \\
\hline Luminal A & 193 & 28.7 & 459 & 50.8 & 652 & 41.4 & $<0.001$ \\
\hline Luminal B & 127 & 18.9 & 260 & 28.8 & 387 & 24.6 & \\
\hline HER2-enriched & 40 & 5.9 & 32 & 3.5 & 72 & 4.6 & \\
\hline HR-positive & 1,025 & 69.6 & 1,937 & 88.2 & 2,962 & 80.8 & $<0.001$ \\
\hline TNBC & 448 & 30.4 & 258 & 11.8 & 706 & 19.2 & \\
\hline Total & 1,473 & 40.2 & 2195 & 59.8 & 3,668 & 100 & \\
\hline
\end{tabular}

${ }^{a}$ Chi-square test for differences in proportions, Kruskalis-Wallis and Wilcoxon rank sum test with continuity correction, where appropriate, for continuous variables (median comparisons).

Pts patients, HR hormone receptors, IQR interquartile range, IHC immunohistochemical, TILs tumor-infiltrating lymphocytes.

in a blinded fashion. Overall, 35 discordant cases (35\%) were observed. The discordances were between IHC $1+$ vs. $0(n=15)$, $1+$ vs. $2+(n=12), 2+$ vs. $0(n=1), 3+$ vs. $1+(n=1)$, and $3+$ vs. $2+(n=6)$ scores. In most cases ( 25 of $35,71.4 \%)$, only one pathologist was discordant with the others. The multi-rater overall kappa concordance score was $0.79(p<0.001)$, which is considered a substantial agreement. The kappa scores according to the HER2 IHC categories $0,1+, 2+$, and $3+$ were 0.82 (almost perfect agreement), 0.67 (substantial agreement), 0.74 (substantial agreement) and 0.92 (almost perfect agreement), respectively $(p<$ $0.001)$. Similar results were obtained when the HER2 $3+$ cases were removed (data not shown).

\section{Distribution of the PAM50 intrinsic subtypes}

PAM50 intrinsic subtypes were available from 1,576 (42.7\%) patients. Intrinsic subtypes were differentially distributed among the three IHC-based groups, as well as between HER2-low and HER2 0 tumors $(p<0.001$ for both) (Fig. 3, Table 1, and Supplementary Table 1). Intrinsic subtypes distribution varied also between HR-positive and TNBC $(p<0.001)$ (Fig. 3 and Supplementary Table 2). Specifically, Luminal A tumors were more frequent within the IHC $2+(54.2 \%)$, HER2-low $(50.8 \%)$ and HRpositive (56.6\%) groups compared to IHC $1+(49.0 \%)$, IHC 0 (28.7\%) and TNBC (1.6\%). Similarly, Luminal B were more frequent within the IHC 2+(30.2\%), HER2-low (28.8\%) and HR-positive (33.9\%) groups compared to IHC $1+(28.0 \%), \mathrm{IHC} 0(18.9 \%)$ and TNBC (0.2\%); HER2-enriched (HER2-E) were more frequent within the IHC 0 (5.9\%) and TNBC (8.5\%) groups compared to IHC $2+$ (2.8\%), IHC 1+(4.0\%), HER2-low (3.5\%) and HR-positive tumors (3.1\%); Basal-like tumors were mostly concentrated within the IHC $0(43.7 \%)$ and TNBC (84.7\%) groups compared to IHC $2+(9.8 \%)$, IHC 1+ (15.2\%), HER2-low (13.4\%) and HR-positive tumors (3.9\%).

Within HR-positive disease, intrinsic subtypes were differentially distributed between HER2-low and HER2 0 tumors, as well as according to IHC score $(p<0.001$ in both cases; Table 2 and Supplementary Table 3). Specifically, Luminal B and Basal-like subtypes were less frequent in HER2-low compared to HER2 0 (Luminal B: $8.0 \%$ vs. $34.9 \%$; Basal-like: $1.9 \%$ vs. $33.4 \%$ ), while
Luminal A subtype was more frequent in HER2-low compared to HER2 0 (58.9\% vs. $2.8 \%$ ). There was no significant difference in subtype distribution in TNBC according to HER2-low status and IHC score $(p=0.438$ and $p=0.284$, respectively; Table 2 and Supplementary Table 3 ). When comparing HR-positive and TNBC according to the same HER2 IHC score, intrinsic subtypes were significantly differentially distributed, with Basal-like tumors being the predominant subtype in each TNBC/HER2 subset $(85.2 \%$ in HER2 $0,85.4 \%$ in HER2 $1+, 78.4 \%$ in HER2 2+). As expected, Luminal A $(51.8 \%$ in HER2 $0,57.9 \%$ in HER2 $1+, 60.6 \%$ in HER2 2+), followed by Luminal B subtype (34.9\% in HER2 $0,33.1 \%$ in HER2 $1+, 33.8 \%$ in HER2 $2+$ ), were the most frequent in each HRpositive/HER2 subset (Supplementary Table 4).

Finally, we investigated if the distribution of PAM50 subtypes within HER2-low breast cancer differed according to ERBB2 mRNA levels. To approach it, we divided all patients with HER2-negative disease into tertiles (i.e., from low to high: $\mathrm{T} 1, \mathrm{~T} 2$, and $\mathrm{T} 3$ ) based on ERBB2 expression (Table 3). As expected, subtype distribution differed in HER2-low breast cancer according to ERBB2 levels ( $p<$ 0.001 ) with the T2-3 group being more enriched with Luminal $A$, Luminal B and HER2-E subtypes $(51.5 \%, 34.9 \%$, and $6.3 \%$ ) compared to the T1 group (31.7\%, $15.8 \%$, and $3.6 \%)$. On the contrary, the Basal-like subtype was more frequent in the T1 group compared to the T2-3 group (44.6\% vs $2.9 \%)$. The results were similar when comparing either ERBB2 high/HER2-low and ERBB2 low/HER2-low tumors with the whole HER2-low population $(p<$ 0.001 both) (Table 3$)$.

PAM50 and individual gene expression analyses

PAM50 and individual gene expression data was available in 1,320 (35.8\%) patients. The full list of genes and subtypes' signatures evaluated for differential expression analyses in the overall HER2negative population and according to HR status are reported in Supplementary Table 5.

In the overall population, 34 of 55 genes $(61.8 \%)$ were found differentially expressed between HER2-low and HER2 0 (falsediscovery rate $[\mathrm{FDR}]<5 \%$ ) (Table 4, Supplementary Table 6 and Supplementary Fig. 1). Specifically, 14 genes (41.2\%) were found 
A

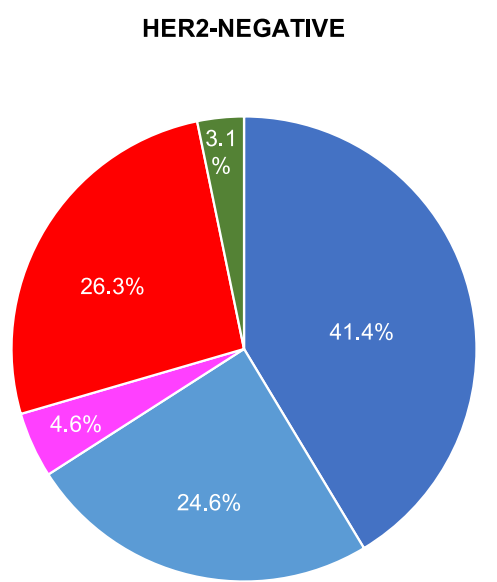

D

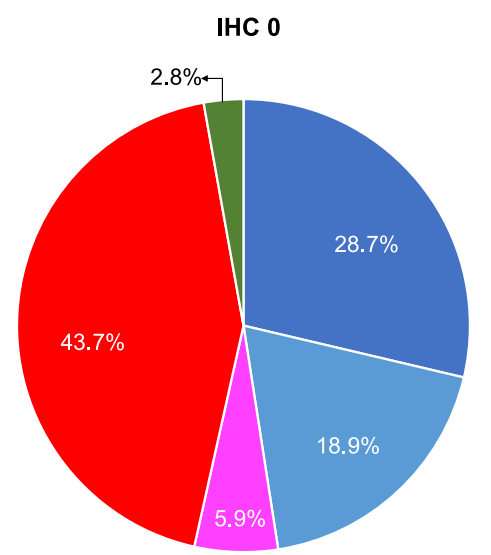

B

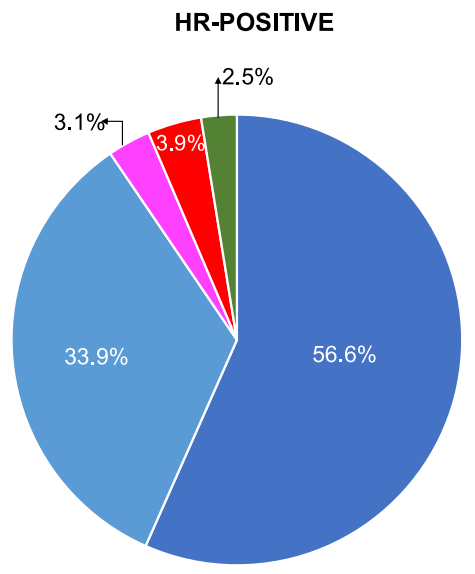

E

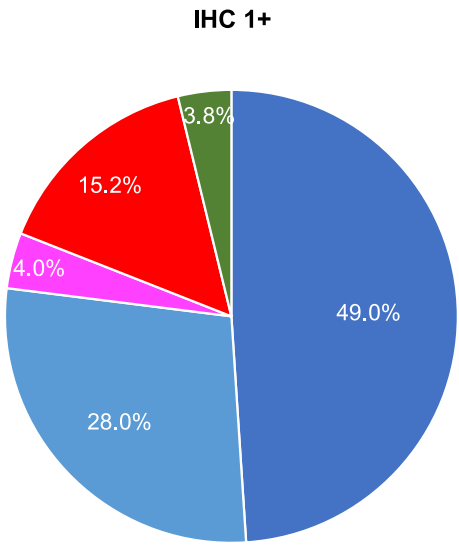

C

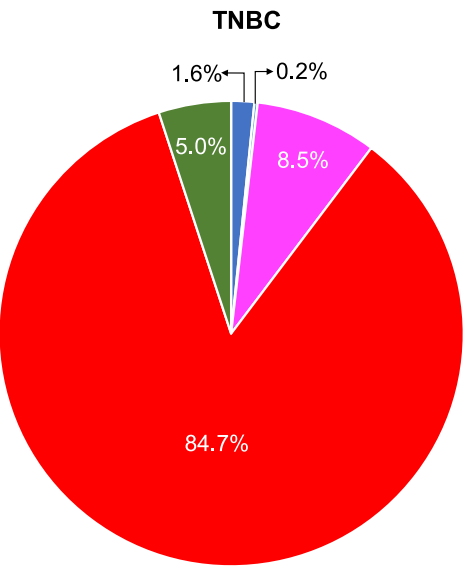

$\mathbf{F}$

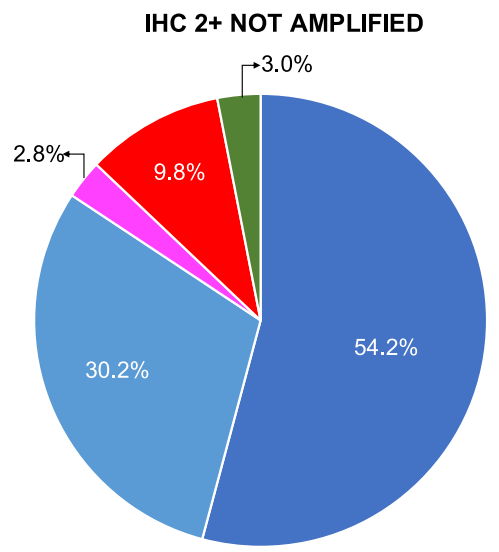

\section{- Luminal A Luminal B HER2-enriched Basal-like $\quad$ Normal-like}

Fig. 3 Intrinsic subtype distribution according to HER2 status and HR status. HR hormone receptors, TNBC triple-negative breast cancer, IHC immunohistochemistry, ISH in situ hybridization (including either FISH, SISH, and CISH). Number of patients in A $(n=1576)$, B $(n=1137)$; $\mathbf{C}(n=437) ; \mathbf{D}(n=673) ; \mathbf{E}(n=701) ; \mathbf{F}(n=325)$.

significantly downregulated in HER2-low compared to HER2 0 , including proliferation-related genes (e.g., CCNB1, CCNE1, MELK, MKI67, MYBL2 etc.), Basal-like-related genes (e.g., KRT14, KRT17, KRT5, FOXC1, MYC etc.), tyrosine-kinase receptors (i.e., EGFR, FGFR4), and three PAM50 signatures (i.e., HER2-E, Basal-like and Normal-like). Conversely, 20 genes (58.8\%) were found significantly upregulated in HER2-low compared to HER2 0 , including luminal-related genes (e.g., BCL2, BAG1, FOXA1, ESR1, PGR, GPR160 and $A R$ ) and two PAM50 signatures (i.e., Luminal $A$ and $B$ ). According to HR status, similar findings were observed in HRpositive disease as in the general population (Table 4, Supplementary Table 6, and Supplementary Fig. 2). In TNBC, however, no individual gene, or PAM50 signature, was found differentially expressed between HER2-low and HER2 0 . Similar findings were observed when HER2-low disease was subdivided into $1+$ and $2+$ (Table 4, Supplementary Table 6, and Supplementary Fig. 3).

Gene expression profiles according to HER2 expression and HR status

The previous results suggested that HR status is a key determinant of the underlying biology of HER2-low breast cancer. To further explore this, we evaluated the overall gene expression profile of HER2-negative breast cancer according to HER2 expression (i.e.,
HER2 $0,1+$ and $2+$ ) and HR status (i.e., positive and negative). The result clearly shows that HR status is the main driver of the underlying biology (Fig. 4 and Supplementary Table 7). As expected, proliferation-related genes (e.g., CCNE1, MKI67 and EXO1) were found more expressed in TNBC compared to HRpositive, regardless of HER2 IHC status (i.e., HER2-low vs. HER2 0). On the contrary, luminal-related genes (e.g., ESR1, $A R$, and $B C L 2$ ) and $E R B B 2$ were found more expressed in HR-positive compared to TNBC, regardless of HER2 IHC status. Of note, the highest ERBB2 expression was found in the HR-positive/HER2-low group. Finally, concordant with the previous results, HER2-low tumors within HRpositive disease showed a relatively lower expression of proliferation-related genes and higher expression of luminalrelated genes compared to the HER2 0 group (Supplementary Fig. 4 and Supplementary Table 8).

\section{ERBB2 expression analysis}

The previous observation that ERBB2 levels differ according to HER2 IHC expression (HER2 $0,1+$, and 2+) and HR status was somewhat unexpected. To further explore this finding, we formally compared the abundance of ERBB2 in HR-positive disease and TNBC based on HER2 IHC expression. ERBB2 levels were statistically significantly higher in HR-positive tumors compared 
Table 2. PAM50 intrinsic subtypes distribution within HR-positive and TN tumors according to HER2 status.

\begin{tabular}{|c|c|c|c|c|c|c|c|}
\hline Luminal A & 187 & 51.8 & 457 & 58.9 & 644 & 56.6 & \multirow[t]{6}{*}{$<0.001$} \\
\hline Luminal B & 126 & 34.9 & 259 & 33.4 & 385 & 33.9 & \\
\hline HER2-enriched & 12 & 3.3 & 23 & 3.0 & 35 & 3.1 & \\
\hline Normal-like & 7 & 1.9 & 22 & 2.8 & 29 & 2.6 & \\
\hline Total & 361 & 31.8 & 776 & 100.0 & 1,137 & 100.0 & \\
\hline \multicolumn{7}{|l|}{ TNBC } & \\
\hline PAM50 subtypes & & & & & & & \\
\hline Luminal A & 5 & 1.6 & 2 & 1.6 & 7 & 1.6 & \multirow[t]{3}{*}{0.438} \\
\hline Luminal B & 1 & 0.3 & 0 & 0.0 & 1 & 0.2 & \\
\hline Total & 311 & 71.2 & 126 & 100.0 & 437 & 100.0 & \\
\hline
\end{tabular}

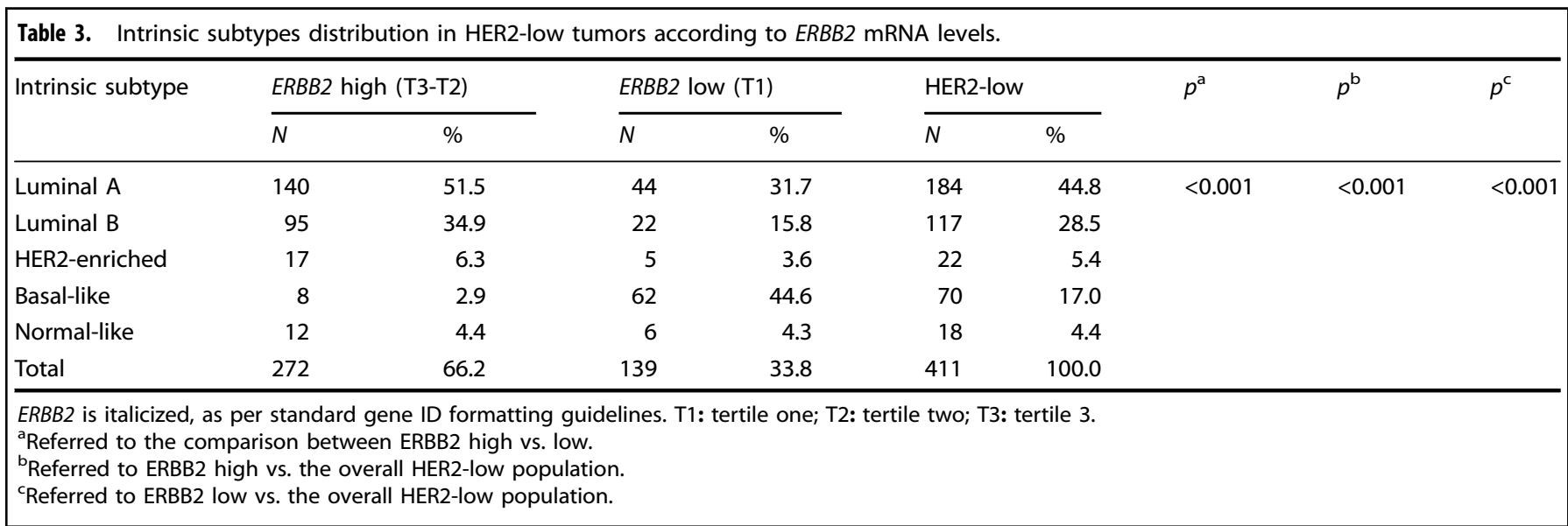

to TNBC regardless of HER2 IHC expression ( $p<0.001$; Fig. 5A, B). Within HR-positive disease, ERBB2 levels were significantly higher in HER2-low tumors compared to HER2 0 (1.4-fold mean difference, $p<0.001$, Fig. 5C), with the highest amount observed in HER2 IHC $2+$ tumors, followed by $1+$ and 0 (Fig. 5D), in decreasing order (1.7-fold mean difference between HER2 2+vs. HER2 0). Within TNBC, there was no statistically significantly difference in ERBB2 levels across the three HER2 IHC groups ( $p=$ 0.080, Fig. 5E); however, TNBC/HER2-low tumors showed statistically significantly higher levels of ERBB2 compared to HER2 0 tumors $(p=0.027)$, although the absolute mean difference was very small (Fig. $5 \mathrm{~F})$.

Prognosis of HER2-low in advanced HER2-negative breast cancer We conducted an exploratory overall survival (OS) analysis in 1,304 patients with advanced breast cancer across two datasets (i.e., Memorial Sloan Kettering Cancer Center database ${ }^{18}$ and Hospital Clinic internal database). OS was defined from the date of the first diagnosis of breast cancer. The median follow-up for the overall population was 90.3 months $(95 \%$ confidence interval $[\mathrm{Cl}]$ : 84.6-99.4). In all patients, no statistically significantly differences in OS were observed between the HER2-low and HER2 0 groups $(p=0.787)$. Similar results were obtained according to HR status and HER2 IHC levels (Fig. 6).

\section{DISCUSSION}

Our results provide preliminary insights of the clinical and molecular characteristics of HER2-low breast cancer. According to our results, patients with HER2-low disease represent the vast majority (59.7\%) of patients with HER2-negative tumors. Clinically, HER2-low breast cancer is apparently more frequent in older and male patients and shows more axillary lymph-node involvement compared to HER2 0 disease. Importantly, we observed that HR status has an important role in HER2-low disease. For example, the frequency of HER2-low disease is higher in HR-positive breast cancer than TNBC (65.4\% vs. $36.6 \%)$ and most HER2-low tumors 
Table 4. Top 20 differentially expressed genes between HER2-low and HER2 0 disease.

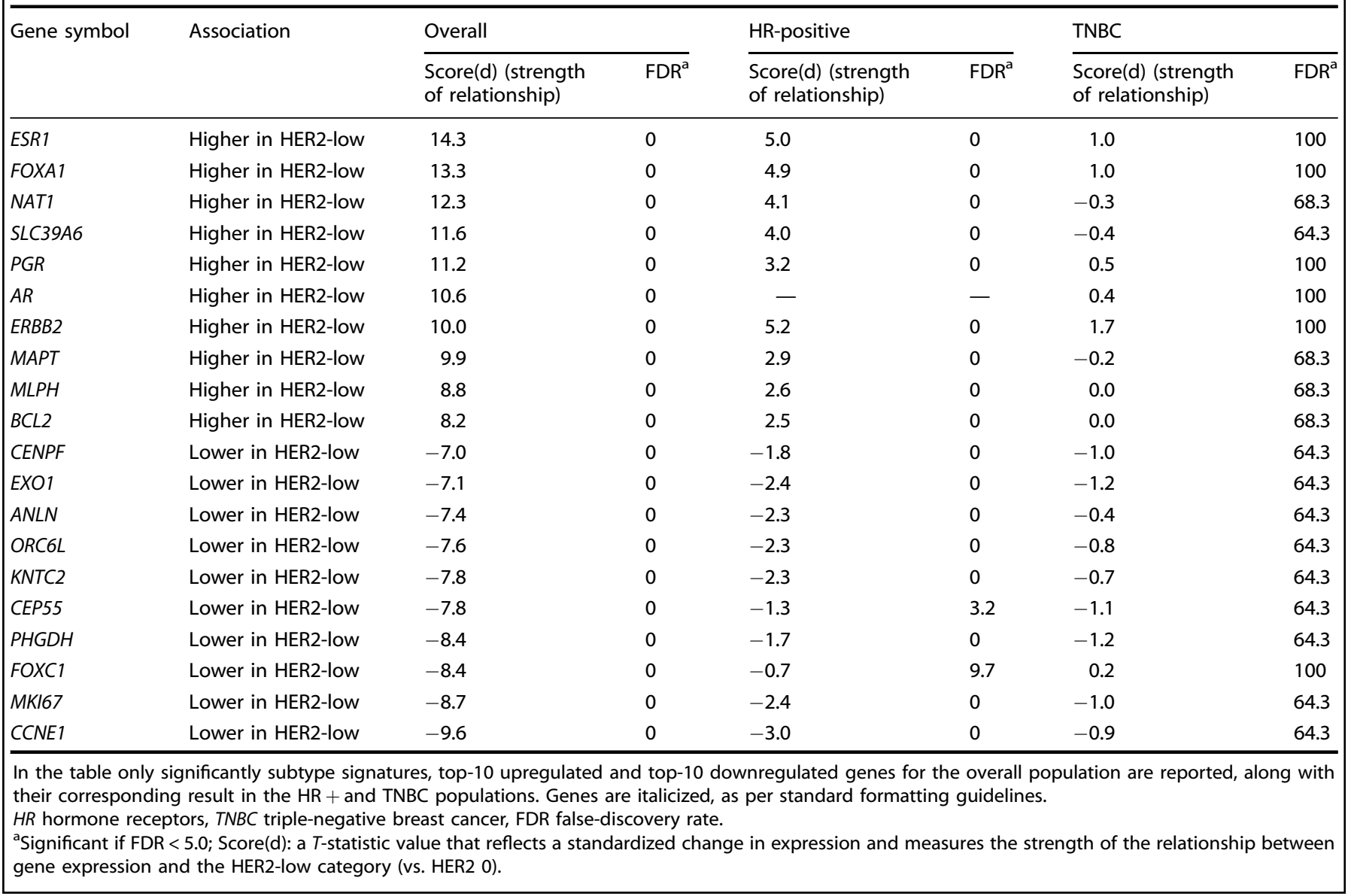

are HR-positive (88.2\%) or Luminal A or B (79.6\%). Another important result of our study is that the vast majority $(67.6 \%)$ of HER2-low tumors have an IHC 1+ score, regardless of HR status. Interestingly, when HR-positive disease and TNBC are divided according to the HER2 IHC score, no significant difference in subtype distribution is observed in TNBC, which was characterized by a high prevalence of the Basal-like subtype (84.7\%), followed by the HER2-E (8.5\%) subtype. On the contrary, HR-positive/HER2-low tumors appeared to be characterized by a higher proportion of luminal subtypes compared to HER2 0 tumors. Of note, the HER2-E subtype was infrequent and similarly distributed in HER2-low and HER2 0 breast cancer.

As expected, the differences in subtype distribution according to HER2 IHC expression and HR status are consistent with the observed changes in expression of individual genes. For example, the vast majority of proliferation-related genes and tyrosine-kinase receptor genes are found more expressed in HER2 0 tumors compared to HER2-low tumors, while HER2-low tumors have more expression of luminal-related genes. This finding is especially relevant in HR-positive disease. On the contrary, no clear biological differences are observed in TNBC according to HER2 IHC expression. Overall, these findings suggest that HR-positive/ HER2-low tumors are a more distinct biological entity compared to TNBC/HER2-low tumors.

The lack of enrichment of the HER2-E subtype within HER2-low disease is intriguing and somewhat unexpected. However, previous studies have shown that the HER2-E phenotype is not defined by the expression of a single gene such as ERBB2. In fact, we and others have previously shown that the two variables (i.e., HER2-E subtype and ERBB2 levels) provide independent predictive and prognostic information ${ }^{19}$. Overall, this finding clearly highlights the need to separate expression of single genes or receptors from the underlying tumor phenotype.

Recent studies have opened up a new therapeutic scenario by showing potent activity of HER2-targeted novel ADCs in HER2-low breast cancer ${ }^{8}$. To date, T-DXd, a trastuzumab conjugated to eight molecules of deruxtecan, a topoisomerase I inhibitor, is at the most advanced in clinical development. A recently published phase Ib study enrolling highly pretreated patients with advanced HER2-expressing/mutated solid tumors, including HER2-low breast cancer, revealed a remarkable overall response rate (ORR) of $37.0 \%$ (95\% Cl: $24.3-51.3 \%)$ in HER2-low breast cancer and an impressive median duration of response of 10.4 months ( $95 \% \mathrm{Cl}$ : 8.8 monthnot evaluable), with no apparent differences in ORR between 1+ and $2+$ IHC tumors $(35.7 \% \text { vs. } 38.5 \%)^{9}$. Interestingly, the ORR did seem to differ according to HR status (40.4\% in HR-positive disease and $14.3 \%$ in TNBC). This result is concordant with our findings that ERBB2 levels are more expresses in HR-positive/HER2-low tumors than in TNBC/HER2-low tumors. A phase III trial specifically enrolling patients with HER2-low metastatic breast cancer (i.e., NCT03734029/DESTINY-Breast04) is ongoing. Importantly, we previously demonstrated in HER2-positive disease that ERBB2 mRNA levels might provide a better selection of patients that benefit to the ADC T-DM $1^{20}$. This might also be the case for HER2low tumors and might be worth focusing on this aspect in further studies.

SYD985 is another ADC comprises trastuzumab covalently bound to a linker drug containing duocarmycin. This drug also showed a promising ORR of 28 and $40 \%$ in HR-positive/HER2-low and TNBC/HER2-low, respectively ${ }^{21}$. In addition, other anti-HER2 


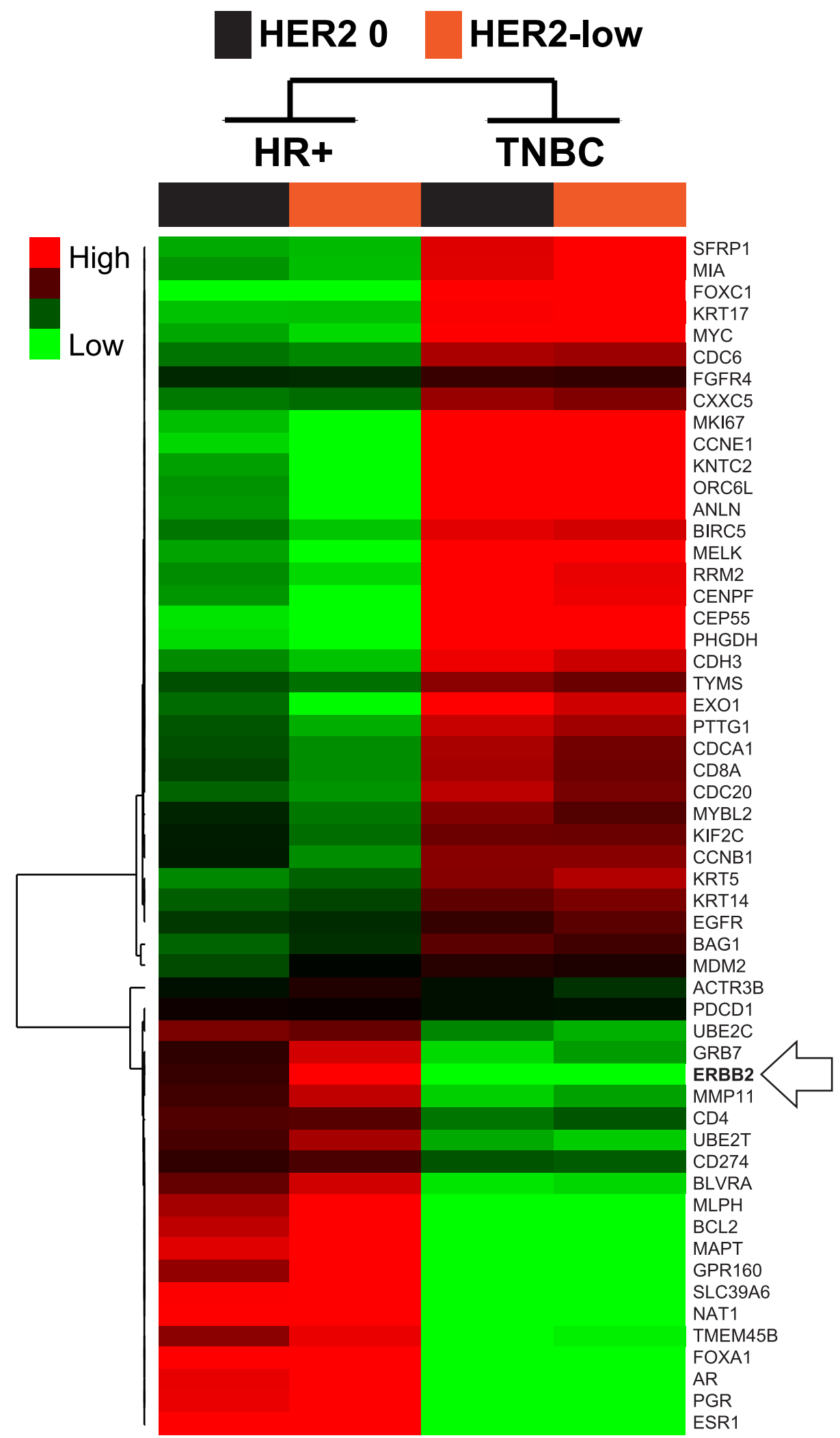

Fig. 4 Gene expression profiles of HER2-negative breast cancer according to HER2 expression and HR status. Supervised clustering of 55 genes across four tumor classes defined according to HER2 IHC expression and HR status. All samples and gene expression data in each category have been combined into a single group. For each gene in a group, we calculated the standardized mean difference between the gene's expression in that class vs. its overall mean expression in the dataset using a 4-class Significance Analyses of Microarrays. The red color represents relative high gene score, green represents relative low gene score, and black represents median gene score. HR-positive hormone receptor positive, TNBC triple-negative breast cancer. 
A

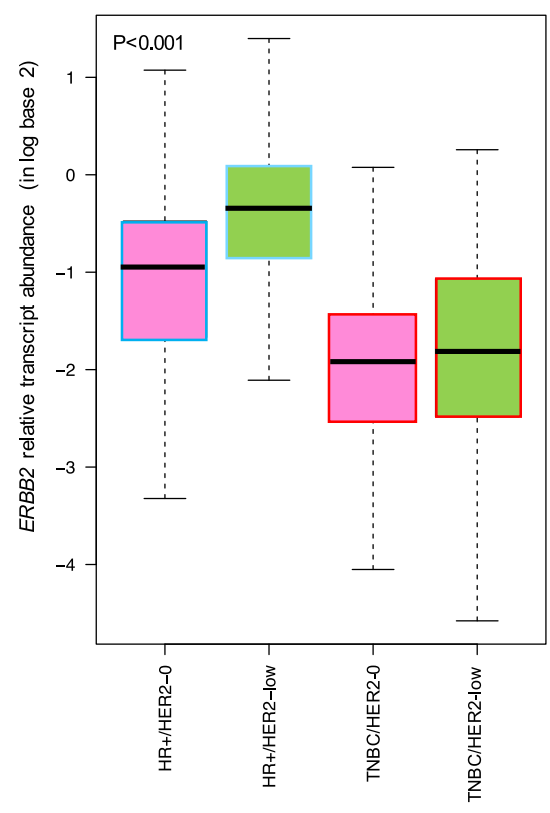

D

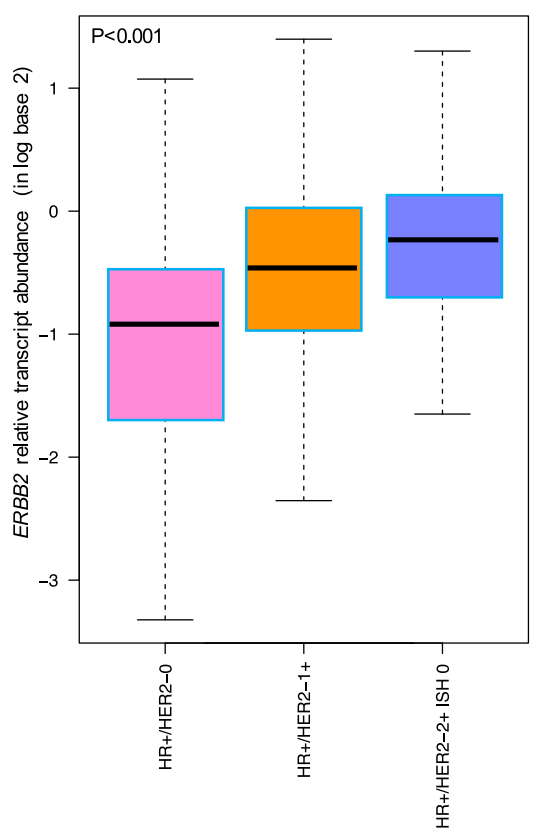

B

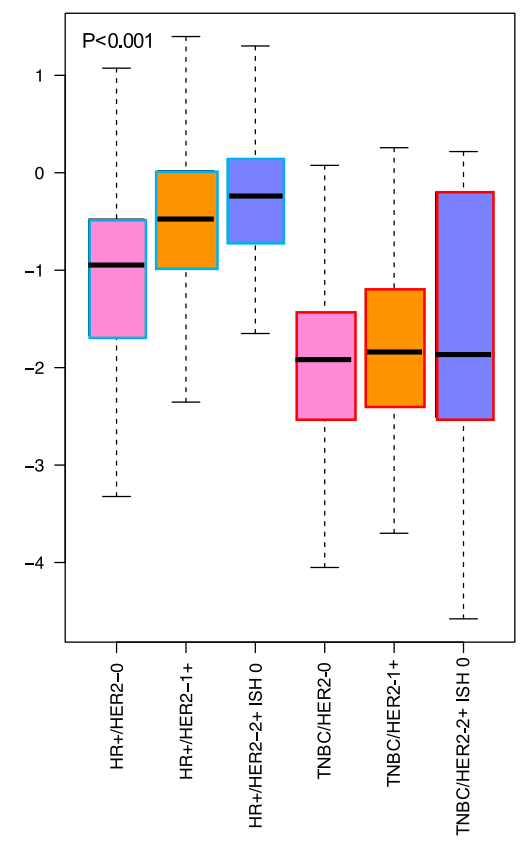

E

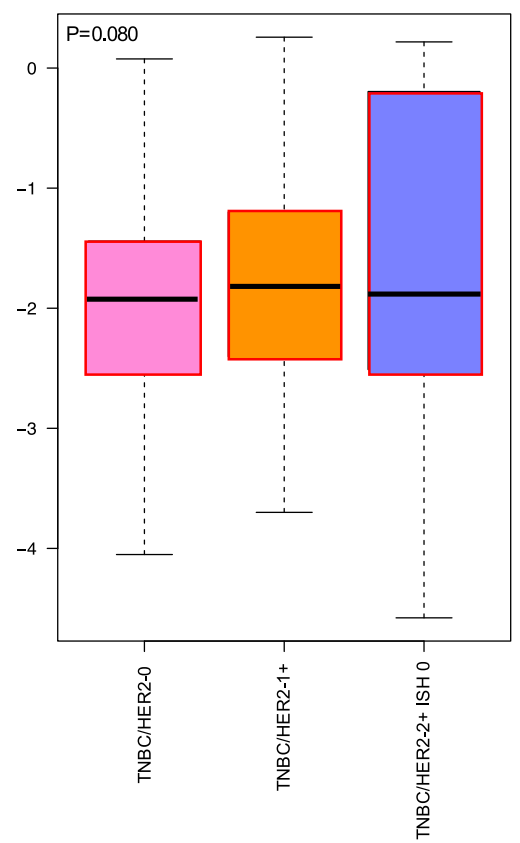

C

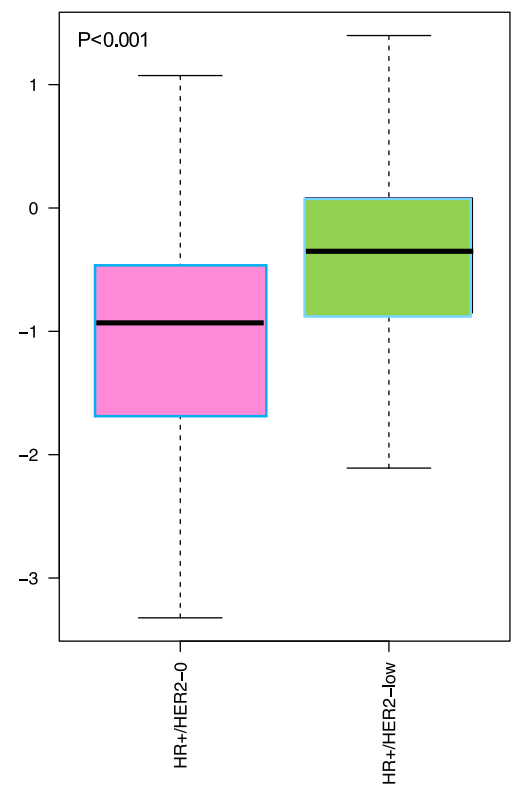

$\mathbf{F}$

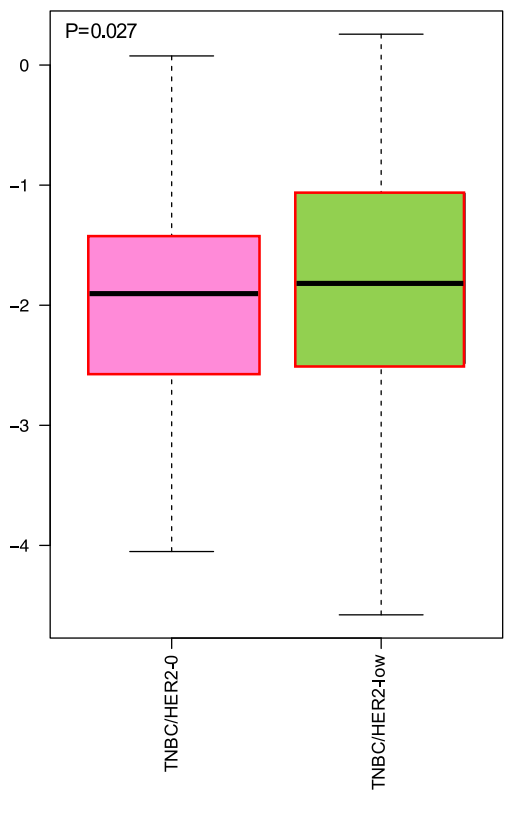

Fig. 5 ERBB2 mRNA levels within the overall, HR-positive and TNBC populations according to HER2-low expression. Relative transcript abundance of ERBB2 (HER2 gene) within the overall population $(n=871)$ and within HR-positive disease $(n=494)$ and TNBC $(n=377)$ according to HER2 IHC-based expression. The boxes represent the interquartile range (25th and 75th percentiles), and the horizontal line in the box represents the median value. The whiskers show the range of largest and smallest values. HR-positive hormone receptor positive, TNBC triple-negative breast cancer.

ADCs (i.e., PF-06804103, MEDI4276, and XMT-1522) have shown promising activity in HER2-low tumors in the preclinical setting ${ }^{8,22}$, and phase 1 clinical trials are ongoing (clinicaltrials.gov identifier: NCT03284723, NCT02564900, and NCT02952729, respectively).

Tumors with high ERBB2 mRNA levels, but overall HER2negative, might also benefit from novel tumor vaccines targeted against the HER2 protein, as shown by a recent randomized phase II trial of HER2-targeted vaccine nelipepimut-S combined with trastuzumab as adjuvant treatment in HER2-low high-risk breast cancer $^{23}$. In this direction, we observed higher levels of TILs in the HER2 2+ group compared to the HER2 0 and 1+groups, although this analysis was based on a very restricted number of cases. Further studies are needed to study the immune compartment of HER2-low breast cancer.

Our study presents limitations that need attention. First, we retrospectively combined patients from databases pertaining to different studies, with different original purposes and inclusion/ exclusion criteria; therefore, patients were not consecutively 
A

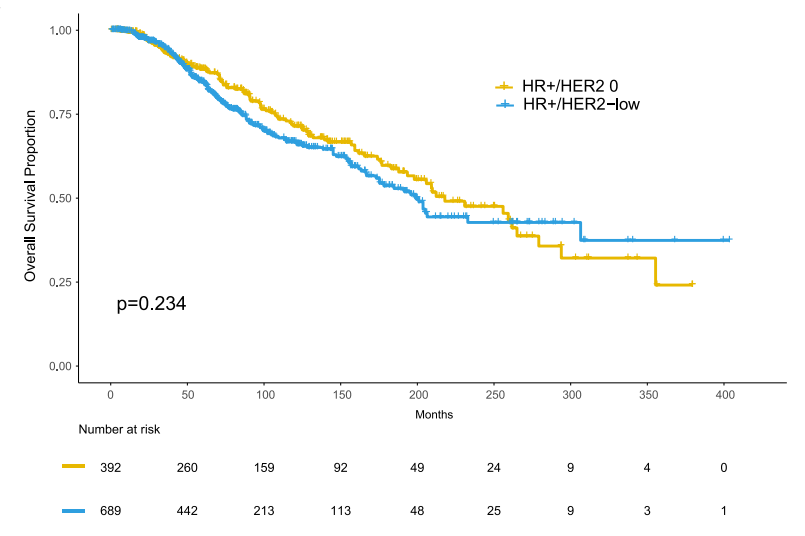

C

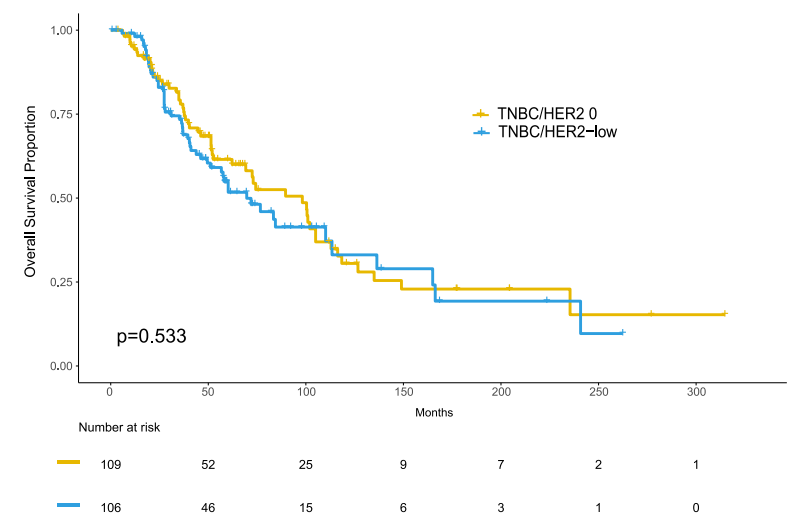

B

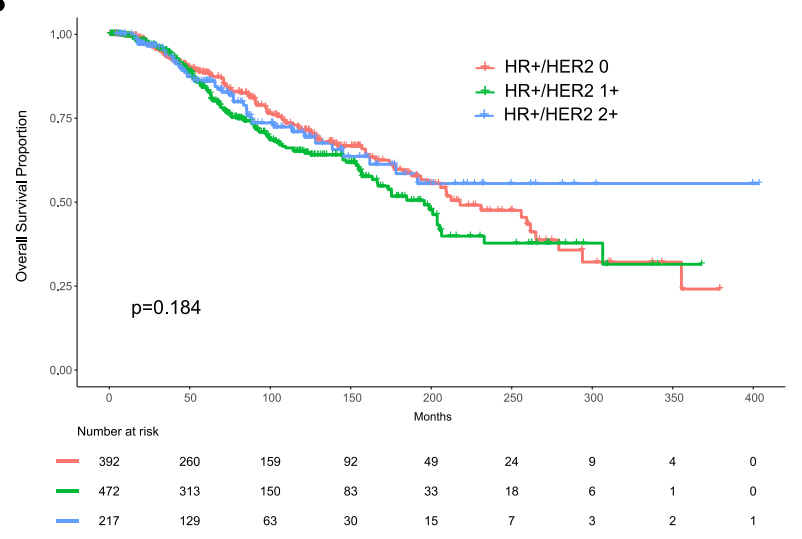

D

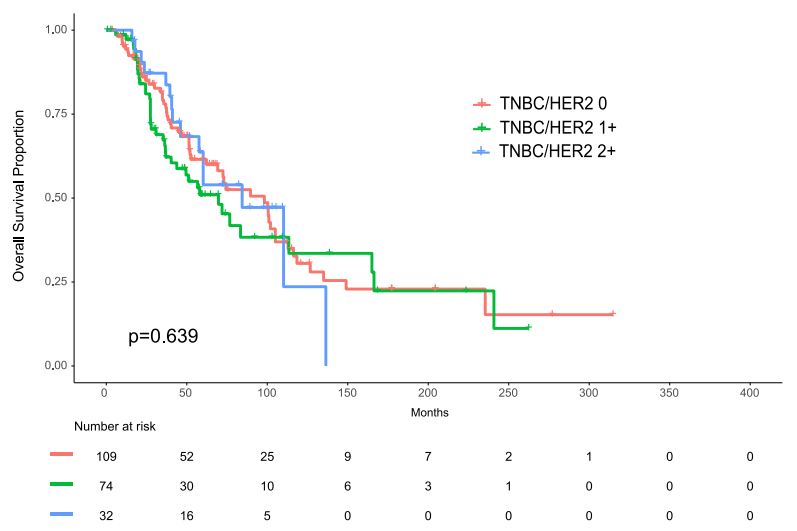

Fig. 6 Overall survival in patients with advanced HER2-negative breast cancer according to HER2 expression. The figure shows Kaplan-Meier curves of overall survival for HER2-low vs HER2 0 tumors in the HR-positive (A) and TNBC (C) populations, as well as OS curves for HER2 2+ vs. HER2 $1+$ vs. HER2 0 tumors for the HR-positive (B) and TNBC (D) populations with number at risk shown at the bottom of each box. $p$-values for log-rank tests are also reported; HR-positive hormone receptor positive, TNBC triple-negative.

enrolled and a large proportion of them had metastatic disease. These might explain some of the imbalances that we observed between groups. Additionally, HER2 IHC status was not evaluated centrally; thus, inter-pathologist variability might have affected the results. Moreover, criteria for defining negative or equivocal ERBB2 amplification have changed over time ${ }^{1,2}$ and most ERBB2 amplification results were only available in qualitative form (i.e., amplified, not amplified or equivocal). Another limitation is that we did not address intra-tumor HER2 heterogeneity, which represents $1 \%-34 \%$ of all breast tumors ${ }^{24}$ and has clinical and prognostic implications, with poor response to anti-HER2-based regimens and worse prognosis, compared to HER2-positive tumors ${ }^{24}$. However, this feature is more common in HER2 equivocal disease ${ }^{24}$, a condition that was an exclusion criteria in our study, somewhat mitigating this issue. Finally, we limited our genomic analysis to the PAM50 genes and five additional genes. Thus, broader genomic analyses are likely to shed more light on this topic.

To our knowledge, this is the first comprehensive study focused specifically on HER2-low breast tumors. We provided extensive comparisons among the three different IHC-based classes of HER2-negative breast cancer and according to HR status. We found that HER2-low breast tumors are complex and heterogeneous, with no specific prognostic implications and HR-positive/ HER2-low emerge as a more distinct biological entity compared to the other groups. In addition, the evidence of ERBB2 levels being higher in HER2-low/HER2 2+ tumors (especially in the HR-positive) compared to HER2 $1+/ 0$ is in line with some previous findings from single institutions-based studies, and contributes to reassure about the reliability of our results ${ }^{25,26}$. Similarly, the high prevalence of luminal disease in HER2-low disease has also been observed in other studies ${ }^{24}$. Finally, the concordance analysis of HER2 scoring by different pathologists showed an almost perfect agreement for HER2 0 and 3+ scores; however, the agreement for the HER2 $1+$ and $2+$ categories was only substantial, according to Landis and Koch interpretation ${ }^{27}$. This result clearly suggests that more efforts are needed to standardize the scoring of HER2-low disease and potentially implement new and more sensitive assays that can help better discriminate HER2 levels within HER2negative breast cancer.

\section{METHODS}

\section{Patients datasets}

All non-overlapping publicly available breast datasets (i.e., 12 studies and 6477 patients) were interrogated from the cBio Cancer Genomics Portal (http://cbioportal.org). From these databases, HER2-negative tumors with known IHC and HER2 amplification status were extracted ${ }^{10-13}$. Other patients were extracted from internal databases from the Hospital Clinic (Barcelona, Spain), from two SOLTI clinical trials (SOLTI 1501-VENTANA and SOLTI 1402-CORALEEN) ${ }^{14,15}$, from the Spanish Cancer Research Group (GEICAM)/CIBOMA study ${ }^{16}$ and from a previously published collaboration between Hospital Clinic (Barcelona, Spain), Hospital Vall d'Hebron (Barcelona, Spain), University Campus Bio-Medico (Roma, Italy) and GEICAM $^{17}$ (see Supplementary Table 9 for study details). All studies had received proper ethical approval by the local institutional research ethics 
committee of all participating institutions and patients had given their consent to participate.

\section{Inclusion criteria}

Patients were included if they were HER2-negative with known IHC and HER2 amplification status and if they had at least one of the following information available: (1) clinicopathological features, (2) PAM50 gene expression data, and (3) PAM50 intrinsic subtype identified. The following clinical-pathological features were evaluated, when available: Ki67 IHC, histological grade, estrogen receptor and progesterone receptor status, age at diagnosis, menopausal status, tumor sample origin (primary vs. metastatic), histological subtype and TILs.

\section{IHC-based classification}

Tumors were divided into HR-positive (i.e., ER and/or $\mathrm{PgR} \geq 1 \%$ ) or TNBC, defined as $\mathrm{ER}<1 \%$ and $\mathrm{PgR}<1 \%$. In addition, tumors were classified into HER2 0 , in case of an IHC score of 0 , and HER2-low, defined as HER2 IHC of $1+$ or $2+$ with an HER2 amplification negative result by in situ hybridization (ISH) techniques. HER2 IHC 0 and $1+$ were considered HER2 0 and HER2-low, respectively, unless ISH-based data was available and reported as HER2-amplified. HER2 status in each cohort had been previously determined using standard FDA-approved antibodies and ISH-techniques and classified according to the ASCO/ CAP guidelines ${ }^{1,2}$. Whenever available, we interpreted ISH-derived HER2/CEP17 ratio value and ERBB2 copy number results jointly with HER2 IHC score, according to last ASCO/CAP guidelines ${ }^{1}$. More specifically, tumors with an average HER2 copy number $<4.0$ signals/ cell, were considered HER2-negative, and also HER2-low in case of an IHC score of $1+$ or $2+$, irrespective of the HER2/CEP17 ratio. However, if the HER2/CEP17 ratio was $\geq 2.0$ and HER2 IHC $3+$, tumors were considered HER2-positive and excluded ${ }^{1}$.

In case of available average HER2 copy number $\geq 4.0$ and $<6.0$ signals/ cell without HER2/CEP ratio and an IHC $3+$, the tumor was considered positive and excluded. In case of $\mathrm{IHC} 0$ or $1+$, the tumor was considered HER2-negative, and also HER2-low in the latter case ${ }^{1}$. In case of IHC 2+, considering the unfeasibility of a retesting, in our case, if the categorization HER2-positive/negative was available from the original dataset, it was adopted and the tumor was considered HER2-negative and HER2-low. If the categorization was not provided, the sample was excluded.

In case of IHC score $0,1+$ or $2+$, and a concurrent average HER2 copy number $\geq 4.0$ and $<6.0$ signals/cell, with HER2/CEP17 ratio $<2.0$, the tumor was considered HER2-negative, and HER2-low in the last two cases. On the contrary, if the HER2/CEP17 watio was $\geq 2.0$, the tumor was considered HER2-positive and excluded ${ }^{1}$.

In case of HER2 copy number $\geq 6.0$ signals/cell, the tumor was considered HER2-positive and excluded in case of IHC of $2+$ or $3+$ regardless of the HER2/CEP17 ratio result, but in case of HER2/CEP17 ratio $<2.0$ and IHC 0 or $1+$, the tumor was considered negative, and also HER2low in the second case'.

Patients with a persistent HER2 equivocal result were excluded ${ }^{1}$.

To evaluate the concordance of the HER2 IHC categories among pathologists, we performed an inter-pathologist concordance analysis across 100 independent cases of HER2 staining (HER2 $0,1+, 2+$, and 3 + ). Five independent breast cancer-specialized pathologists (i.e., BG, ES, $\mathrm{RF}, \mathrm{VP}$, and GP) from four institutions (i.e., Hospital Clinic, VHIO, $\mathrm{HVH}$, and Campus Bio-Medico) were involved. Blinded scores were provided to FS and AP, who performed the concordance analysis.

\section{PAM50 subtypes and gene expression data}

We obtained PAM50 subtype information and individual gene expression data from 9 of the 13 retrospective cohorts (Hospital Clinic internal series, SOLTI and GEICAM trials reported in Supplementary Table 9). An nCounterbased research version of PAM50 had been previously used ${ }^{28,29}$. Intrinsic subtypes and raw gene expression data had been obtained from formalinfixed paraffin-embedded (FFPE) tumor samples. For RNA purification (Roche High Pure FFPET RNA isolation kit), at least 1 to $310-\mu \mathrm{m}$ FFPE slides had been used for each tumor specimen, and macrodissection performed, when needed, to avoid normal breast tissue contamination. A minimum of $\sim 150 \mathrm{ng}$ of total RNA had been used to measure the expression of 50 breast cancer-related genes, 4 immune-related genes, androgen receptor gene (full gene list included in Supplementary Table 5), and 5 housekeeping genes (ACTB, MRPL19, PSMC4, RPLPO, and SF3A1) using the nCounter platform (NanoString Technologies, Seattle WA $)^{28,30}$. Data had been log base 2 transformed and normalized using the five housekeeping genes. Intrinsic subtyping (Luminal A, Luminal B, HER2-E, Basal-like and Normal-like) had been previously performed using the research-based PAM50 intrinsic subtype predictor ${ }^{29}$. We also retrieved intrinsic subtypes from the publicly available TCGA database (see "Data availability" section for further information).

\section{Statistical analysis}

Patient and tumor characteristics were analyzed using chi-square $\left(X^{2}\right)$ test, Fisher's exact test, Kruskalis-Wallis and Wilcoxon rank sum test with continuity correction, where appropriate. The concordance analysis among pathologists was performed using the Fleiss' Kappa. The agreement among pathologists was considered poor for $k<0$, low for $k=0.01-0.20$, fair for $k=0.21-0.40$, moderate for $k=0.41-0.60$, substantial for $k=$ $0.61-0.80$, and almost perfect for $k=0.81-1.00^{27}$.

All differences were considered significant at $p<0.05$. Bonferroni-Holm method was used to control the family-wise error rate in case of multiple comparisons.

OS was evaluated for patients with homogeneous follow-up with available or computable survival data. Such patients pertained to the Memorial Sloan Kettering Cancer Center (MSKCC)'s subset of the cBio Cancer Genomics Portal group and to the Hospital Clinic of Barcelona subset. All patients were affected by metastatic disease and presented available information regarding primary tumor diagnosis.

The OS distributions were estimated using the Kaplan-Meier method and the log-rank test was used to assess the difference in survival distribution between the groups ${ }^{31}$. Censoring was done at the date of last available follow-up. Significance Analysis of Microarray (SAM) for unpaired samples (multiclass and two class) was used to compare gene expression profiles between groups ${ }^{32}$. Differences were considered significant at an $\mathrm{FDR}<5 \%$. All analyses were performed with $\mathrm{R}$ version $3.6 .1^{33}$, Cluster 3.0, Javatreeview 1.1.6r $4^{34}$ and Microsoft Excel.

\section{Reporting summary}

Further information on research design is available in the Nature Research Reporting Summary linked to this article.

\section{DATA AVAILABILITY}

This study involved the collection and analysis of clinicopathological and PAM50 gene expression data from multiple publicly available datasets ${ }^{35-46}$. The following cBioPortal datasets were used: https://identifiers.org/cbioportal:breast_msk_2018; https://identifiers. org/cbioportal:bfn_duke_nus_2015; https://identifiers.org/cbioportal:brca_mskcc_2019; https://identifiers.org/cbioportal:brca_bccrc_xenograft_2014; https://identifiers.org/cbiop ortal:brca_bccrc; https://identifiers.org/cbioportal:brca_broad; https://identifiers.org/cbiop ortal:brca_sanger; https://identifiers.org/cbioportal:brca_tcga; https://identifiers.org/cbiop ortal:brca_igr_2015; https://identifiers.org/cbioportal:brca_metabric; https://identifiers org/cbioportal:brca_mbcproject_wagle_2017; https://identifiers.org/cbioportal:acbc_msk cc_2015. Data from the internal studies of the Hospital Clinic of Barcelona, and data from patients involved in the SOLTI and GEICAM trials included, are not publicly available to protect patient privacy, but will be made available on reasonable request from the corresponding author, Prof. Aleix Prat (email address: alprat@clinic.cat). An anonymized data file containing all PAM50 normalized gene expression data used for the genomic analyses of this study, is publicly available in the figshare repository ${ }^{47}$, with https://doi. org/10.6084/m9.figshare.13171655. The complete version of the data file used and/or analyzed during the current study, is available upon reasonable request from the corresponding author, as described in the figshare data record above.

\section{CODE AVAILABILITY}

$\mathrm{R}$ codes are available from the corresponding author on reasonable request.

Received: 30 April 2020; Accepted: 13 November 2020; Published online: 04 January 2021

\section{REFERENCES}

1. Wolff, A. C. et al. Human epidermal growth factor receptor 2 testing in breast cancer: American Society of Clinical Oncology/College of American Pathologists Clinical Practice Guideline Focused Update. J. Clin. Oncol. J. Am. Soc. Clin. Oncol. 36, 2105-2122 (2018). 
2. Wolff, A. C. et al. Recommendations for human epidermal growth factor receptor 2 testing in breast cancer: American Society of Clinical Oncology/College of American Pathologists clinical practice guideline update. J. Clin. Oncol. J. Am. Soc. Clin. Oncol. 31, 3997-4013 (2013).

3. Cronin, K. A., Harlan, L. C., Dodd, K. W., Abrams, J. S. \& Ballard-Barbash, R. Population-based estimate of the prevalence of HER-2 positive breast cancer tumors for early stage patients in the US. Cancer Invest. 28, 963-968 (2010).

4. Slamon, D. J. et al. Human breast cancer: correlation of relapse and survival with amplification of the HER-2/neu oncogene. Science 235, 177-182 (1987).

5. Schalper, K. A., Kumar, S., Hui, P., Rimm, D. L. \& Gershkovich, P. A retrospective population-based comparison of HER2 immunohistochemistry and fluorescence in situ hybridization in breast carcinomas: impact of 2007 American Society of Clinical Oncology/College of American Pathologists criteria. Arch. Pathol. Lab. Med. 138, 213-219 (2014).

6. Fehrenbacher, L. et al. NSABP B-47/NRG oncology phase III randomized trial comparing adjuvant chemotherapy with or without trastuzumab in high-risk invasive breast cancer negative for HER2 by FISH and with IHC 1+ or 2. J. Clin. Oncol. J. Am. Soc. Clin. Oncol. 38, 444-453 (2020).

7. Iwata, H. et al. Trastuzumab deruxtecan (DS-8201a) in subjects with HER2expressing solid tumors: long-term results of a large phase 1 study with multiple expansion cohorts. J. Clin. Oncol. 36, 2501-2501 (2018).

8. Rinnerthaler, G., Gampenrieder, S. P. \& Greil, R. HER2 directed antibody-drugconjugates beyond T-DM1 in breast cancer. Int. J. Mol. Sci. 20, 1115 (2019). https://doi.org/10.3390/ijms20051115.

9. Modi, S. et al. Antitumor activity and safety of trastuzumab deruxtecan in patients with HER2-low-expressing advanced breast cancer: results from a phase lb study. J. Clin. Oncol. Off. J. Am. Soc. Clin. Oncol. JCO1902318-JCO1902318, https://doi. org/10.1200/JCO.19.02318 (2020).

10. Cancer Genome Atlas Network. Comprehensive molecular portraits of human breast tumours. Nature 490, 61-70 (2012).

11. Ciriello, G. et al. Comprehensive molecular portraits of invasive lobular breast cancer. Cell 163, 506-519 (2015).

12. The Metastatic Breast Cancer Project. https://www.mbcproject.org/ (2019).

13. Razavi, P. et al. The genomic landscape of endocrine-resistant advanced breast cancers. Cancer Cell 34, 427-438.e6 (2018).

14. Adamo, B. et al. Oral metronomic vinorelbine combined with endocrine therapy in hormone receptor-positive HER2-negative breast cancer: SOLTI-1501 VENTANA window of opportunity trial. Breast Cancer Res. BCR 21, 108 (2019).

15. Prat, A. et al. Ribociclib plus letrozole versus chemotherapy for postmenopausal women with hormone receptor-positive, HER2-negative, luminal B breast cancer (CORALLEEN): an open-label, multicentre, randomised, phase 2 trial. Lancet Oncol. 21, 33-43 (2020).

16. Lluch, A. et al. Phase III trial of adjuvant capecitabine after standard neo-/adjuvant chemotherapy in patients with early triple-negative breast cancer (GEICAM/200311_CIBOMA/2004-01). J. Clin. Oncol. J. Am. Soc. Clin. Oncol. 38, 203-213 (2020).

17. Fernandez-Martinez, A. et al. Limitations in predicting PAM50 intrinsic subtype and risk of relapse score with Ki67 in estrogen receptor-positive HER2-negative breast cancer. Oncotarget 8, 21930-21937 (2017).

18. Cerami, E. et al. The cBio cancer genomics portal: an open platform for exploring multidimensional cancer genomics data. Cancer Discov. 2, 401-404 (2012).

19. Prat, A. et al. HER2-enriched subtype and ERBB2 expression in HER2-positive breast cancer treated with dual HER2 blockade. J. Natl. Cancer Inst. https://doi. org/10.1093/jnci/djz042 (2019).

20. Griguolo, G. et al. ERBB2 mRNA expression and response to ado-trastuzumab emtansine (T-DM1) in HER2-positive breast cancer. Cancers (Basel) 12, 1902 (2020).

21. Banerji, U. et al. Trastuzumab duocarmazine in locally advanced and metastatic solid tumours and HER2-expressing breast cancer: a phase 1 dose-escalation and dose-expansion study. Lancet Oncol. 20, 1124-1135 (2019).

22. Graziani, E. I. et al. PF-06804103, a site-specific anti-HER2 antibody-drug conjugate for the treatment of HER2-expressing breast, gastric, and lung cancers. Mol. Cancer Ther. molcanther.0237.2020, https://doi.org/10.1158/1535-7163.MCT20-0237 (2020).

23. Clifton, G. T. et al. Results of a randomized phase llb trial of nelipepimut-S + trastuzumab vs trastuzumab to prevent recurrences in high-risk HER2 lowexpressing breast cancer patients. Clin. Cancer Res. Off. J. Am. Assoc. Cancer Res. https://doi.org/10.1158/1078-0432.CCR-19-2741 (2020).

24. Marchiò, C. et al. Evolving concepts in HER2 evaluation in breast cancer: Heterogeneity, HER2-low carcinomas and beyond. Semin. Cancer Biol. https://doi.org/ 10.1016/j.semcancer.2020.02.016 (2020).

25. Marchiò, C. et al. The dilemma of HER2 double-equivocal breast carcinomas: genomic profiling and implications for treatment. Am. J. Surg. Pathol. 42, 1190-1200 (2018).

26. Gupta, S. et al. Quantitative assessments and clinical outcomes in HER2 equivocal 2018 ASCO/CAP ISH group 4 breast cancer. NPJ Breast Cancer 5, 28-28 (2019).
27. Landis, J. R. \& Koch, G. G. The measurement of observer agreement for categorical data. Biometrics 33, 159-174 (1977).

28. Geiss, G. K. et al. Direct multiplexed measurement of gene expression with colorcoded probe pairs. Nat. Biotechnol. 26, 317-325 (2008).

29. Prat, A. et al. Prognostic value of intrinsic subtypes in hormone receptor-positive metastatic breast cancer treated with letrozole with or without lapatinib. JAMA Oncol. 2, 1287-1294 (2016).

30. Prat, A., Ellis, M. J. \& Perou, C. M. Practical implications of gene-expression-based assays for breast oncologists. Nat. Rev. Clin. Oncol. 9, 48-57 (2011).

31. Armitage, P., Berry, G. \& Matthews, J. N. S. Statistical Methods in Medical Research (4th edn) (Blackwell Science, Oxford, 2001).

32. Schwender, H. R: Significance Analysis of Microarray. http://ugrad.stat.ubc.ca/R/ library/siggenes/html/sam.html (2006).

33. $\mathrm{R}$ Core Team. R: A language and environment for statistical computing. (R Foundation for Statistical Computing, 2017).

34. Eisen, M. B., Spellman, P. T., Brown, P. O. \& Botstein, D. Cluster analysis and display of genome-wide expression patterns. Proc. Natl Acad. Sci. USA 95, 14863-14868 (1998).

35. Razavi, P. et al. Targeted sequencing of tumor/normal sample pairs from 1918 Breast cancers. The cBioPortal for Cancer Genomics https://identifiers.org/ cbioportal:breast_msk_2018 (2018).

36. Tan, J. et al. Whole exome sequencing of 22 phyllodes tumors. The cBioPortal for Cancer Genomics https://identifiers.org/cbioportal:bfn_duke_nus_2015 (2015).

37. Nixon, J. M. et al. Targeted Sequencing of buparlisib+letrozole and alpelisib + letrozole-treated metastatic ER+ unmatched breast tumors. The CBioPortal for Cancer Genomics. https://identifiers.org/cbioportal:brca_mskcc_2019 (2019).

38. Eirew, P. et al. Whole genome/targeted sequencing to evaluate the clonal dynamics in 116 breast cancer patient xenografts. The cBioPortal for Cancer Genomics. https://identifiers.org/cbioportal:brca_bccrc_xenograft_2014 (2015).

39. Shah, P. S. et al. Whole genome/exome sequencing analysis of 65 breast cancer samples. The CBioPortal for Cancer Genomics. https://identifiers.org/cbioportal: brca_bccrc (2012).

40. Banerji, S. et al. Whole-exome sequencing of 103 breast cancer tumor/normal sample pairs. Generated by the Broad Institute. The cBioPortal for Cancer Genomics. https://identifiers.org/cbioportal:brca_broad (2012).

41. Stephens, J. P. et al. Whole exome sequencing from 100 breast cancer tumor/ normal sample pairs. Generated by the Sanger Institute. The cBioPortal for Cancer Genomics. https://identifiers.org/cbioportal:brca_sanger (2012).

42. TCGA Breast Invasive Carcinoma. Source data from GDAC Firehose. Previously known as TCGA Provisional. The cBioPortal for Cancer Genomics https://identifiers. org/cbioportal:brca_tcga (2016).

43. Lefebvre, C. et al. Whole exome sequencing of 216 tumor/normal (blood) pairs from metastatic breast cancer patients who underwent a biopsy in the context of the SAFIR01/SAFIR02 (Unicancer, France), SHIVA (Institut Curie, France) or MOSCATO (Gustave Roussy, France) prospective trials. The cBioPortal for Cancer Genomics. https://identifiers.org/cbioportal:brca_igr_2015 (2016).

44. Pereira, B. et al. Targeted sequencing of 2509 primary breast tumors with 548 matched normal. The cBioPortal for Cancer Genomics https://identifiers.org/ cbioportal:brca_metabric (2016).

45. The Metastatic Breast Cancer Project (Provisional, February 2020). The cBioPortal for Cancer Genomics https://identifiers.org/cbioportal:brca_mbcproject_wagle_ 2017 (2020).

46. Martelotto, G. L. et al. Whole exome sequencing of 12 breast AdCCs. The cBioPortal for Cancer Genomics https://identifiers.org/cbioportal:acbc_mskcc_2015 (2015).

47. Schettini, F. et al. Data and metadata supporting the article: Clinical, pathological and PAM50 gene expression features of HER2-low breast cancer. figshare https:// doi.org/10.6084/m9.figshare.13171655 (2020).

\section{ACKNOWLEDGEMENTS}

This work was supported by the grants from the European Union's Horizon 2020 Research and Innovation Programme under Grant agreement No. 847912 (to A.P.), the Instituto de Salud Carlos III-PI16/00904 (to A.P.), Pas a Pas (to A.P.), Save the Mama (to A.P.), Breast Cancer Now-2018NOVPCC1294 (to A.P.), Fundación Científica Asociación Española Contra el Cáncer-Ayuda Postdoctoral AECC 2017 (to F.B.-M.), Fundación SEOM, Becas FSEOM para Formación en Investigación en Centros de Referencia en el Extranjero 2018 (to T.P.) and PhD4MD - Departament de Salut expedient SLT008/18/00122 (to N.C.).

\section{AUTHOR CONTRIBUTIONS}

F.S. and A.P. conceived the study. F.S. and L.P. performed the statistical analyses. All authors contributed to the interpretation of results, writing and/or critical revision of the manuscript and approved the final version. 


\section{COMPETING INTERESTS}

A.P. has declared an immediate family member being employed by Novartis, personal honoraria from Pfizer, Novartis, Roche, MSD Oncology, Lilly and Daiichi Sankyo, travel, accommodations and expenses paid by Daiichi Sankyo, research funding from Roche and Novartis, consulting/advisory role for NanoString Technologies, Amgen, Roche, Novartis, Pfizer and Bristol-Myers Squibb, and patent PCT/EP2016/080056: HER2 AS A PREDICTOR OF RESPONSE TO DUAL HER2 BLOCKADE IN THE ABSENCE OF CYTOTOXIC THERAPY. C.B. declares research funding, consulting and honoraria form Astra Zeneca, Novartis, Roche, GSK, Pfizer, Libbs, Daiichi Sankyo, and MSD. A.L. declares clinical research fundings from Amgen, Astra Zeneca, Boehringer-Ingelheim, GSK, Novartis, Pfizer, Roche/Genentech, Eisai, Celgene, Pierre Fabre and advisory boards and consulting for Novartis, Pfizer, Roche/Genentech, Eisai, Celgene. M.M. declares research grants from Roche, PUMA and Novartis, consulting/advisory fees from AstraZeneca, Amgen, Taiho Oncology, Roche/ Genentech, Novartis, PharmaMar, Eli Lilly, PUMA, Taiho Oncology, Daiichi Sankyo and Pfizer and speakers' honoraria from AstraZeneca, Amgen, Roche/Genentech Novartis and Pfizer. J.G. has declared speakers' honoraria and participation in advisory boards from Pfizer, Roche, and Novartis. S.D.P. has declared honoraria from Roche, Pfizer, Astra-Zeneca, Novartis, Celgene, Eli Lilly, Amgen, and Eisai. The other authors have nothing to declare.

\section{ADDITIONAL INFORMATION}

Supplementary information is available for this paper at https://doi.org/10.1038/ s41523-020-00208-2.
Correspondence and requests for materials should be addressed to A.P.

Reprints and permission information is available at http://www.nature.com/ reprints

Publisher's note Springer Nature remains neutral with regard to jurisdictional claims in published maps and institutional affiliations.

Attribution 4.0 International License, which permits use, sharing, adaptation, distribution and reproduction in any medium or format, as long as you give appropriate credit to the original author(s) and the source, provide a link to the Creative Commons license, and indicate if changes were made. The images or other third party material in this article are included in the article's Creative Commons license, unless indicated otherwise in a credit line to the material. If material is not included in the article's Creative Commons license and your intended use is not permitted by statutory regulation or exceeds the permitted use, you will need to obtain permission directly from the copyright holder. To view a copy of this license, visit http://creativecommons. org/licenses/by/4.0/.

c) The Author(s) 2021 